\title{
A Family of Generalized Linear Models for Repeated Measures With Normal and Conjugate Random Effects
}

\author{
Geert Molenberghs $^{1,2} \quad$ Geert Verbeke $^{2,1} \quad$ Clarice G.B. Demétrio $^{3}$ \\ Afrânio M.C. Vieira ${ }^{3,4}$ \\ 1 I-BioStat, Universiteit Hasselt, B-3590 Diepenbeek, Belgium \\ 2 I-BioStat, Katholieke Universiteit Leuven, B-3000 Leuven, Belgium \\ ${ }^{3}$ ESALQ, Piracicaba, Saõ Paulo, Brazil \\ ${ }^{4}$ Universidade de Brasília, Dept. de Estatística, Brasília/DF, Brasil
}

\section{Supplementary Materials}

\section{A Generic Approximations for Marginal Model Elements}

As stated in Section 4.2, in general, an approximate mean can be derived using the expansion, around $\boldsymbol{b}_{\boldsymbol{i}}=\mathbf{0}$,

$$
\kappa_{i j} \approx g\left(\eta_{i j}\right)+g^{\prime}\left(\eta_{i j}\right) \boldsymbol{z}_{i j}^{\prime} \boldsymbol{b}_{\boldsymbol{i}}+\frac{1}{2} g^{\prime \prime}\left(\eta_{i j}\right) \boldsymbol{z}_{i j}^{\prime} \boldsymbol{b}_{\boldsymbol{i}} \boldsymbol{b}_{\boldsymbol{i}}{ }^{\prime} \boldsymbol{z}_{i j},
$$

i.e.,

$$
E\left(Y_{i j}\right) \approx \vartheta_{i j}\left[g\left(\eta_{i j}^{(0)}\right)+\frac{1}{2} g^{\prime \prime}\left(\eta_{i j}^{(0)}\right) \boldsymbol{z}_{i j}^{\prime} D \boldsymbol{z}_{i j}\right],
$$

where $\eta_{i j}^{(0)}=\boldsymbol{x}_{i j}^{\prime} \boldsymbol{\xi}$. A general variance expression can be derived in a similar fashion, based upon:

$$
\operatorname{Var}\left(Y_{i j}\right)=E\left\{E\left[\operatorname{Var}\left(Y_{i j} \mid \boldsymbol{b}_{\boldsymbol{i}}, \theta_{i j}\right)\right]\right\}+E\left\{\operatorname{Var}\left[E\left(Y_{i j} \mid \boldsymbol{b}_{\boldsymbol{i}}, \theta_{i j}\right)\right]\right\}+\operatorname{Var}\left\{E\left[E\left(Y_{i j} \mid \boldsymbol{b}_{\boldsymbol{i}}, \theta_{i j}\right)\right]\right\} .
$$

To simplify ensuing derivations, write the variance function as

$$
\omega\left(\mu_{i j}^{c}\right)=\omega_{i j}\left(\theta_{i j} \kappa_{i j}\right)=\phi \psi^{\prime \prime}\left[h\left(\theta_{i j} \kappa_{i j}\right)\right] .
$$

Note that $\omega(\cdot)$ allows for all of the traditional mean-variance relationships of GLMs for Gaussian, binary, binomial, count, and time-to-event data. Straightforward but tedious algebraic derivations, based on expansions around $\theta_{i j}=1$ and $\boldsymbol{b}_{\boldsymbol{i}}=\mathbf{0}$, leads to:

$$
\begin{aligned}
\operatorname{Var}\left(Y_{i j}\right) \approx & \omega\left[g\left(\eta_{i j}^{(0)}\right)\right]+\omega^{\prime}\left[g\left(\eta_{i j}^{(0)}\right)\right]\left(\vartheta_{i j}-1\right)+\frac{1}{2} \omega^{\prime \prime}\left[g\left(\eta_{i j}^{(0)}\right)\right] g^{2}\left(\eta_{i j}^{(0)}\right)\left(\sigma_{i j}^{2}+\vartheta_{i j}^{2}-2 \vartheta_{i j}+1\right) \\
& +\frac{1}{2} \omega^{\prime \prime}\left[g\left(\eta_{i j}^{(0)}\right)\right] g\left(\eta_{i j}^{(0)}\right) g^{\prime}\left(\eta_{i j}^{(0)}\right)\left(\vartheta_{i j}^{2}+\sigma_{i j}^{2}\right) \boldsymbol{z}_{i j}^{\prime} D \boldsymbol{z}_{i j}+\sigma_{i j}^{2} g^{2}\left(\eta_{i j}^{(0)}\right) \\
& +\frac{1}{2} \omega^{\prime}\left[g\left(\eta_{i j}^{(0)}\right)\right] g^{\prime \prime}\left(\eta_{i j}^{(0)}\right) \vartheta_{i j} \boldsymbol{z}_{i j}^{\prime} D \boldsymbol{z}_{i j}+\xi_{i j}^{2}\left[g^{\prime}\left(\eta_{i j}^{(0)}\right)\right]^{2} \boldsymbol{z}_{i j}^{\prime} D \boldsymbol{z}_{i j} \\
& +\sigma_{i j}^{2}\left[g^{\prime 2}\left(\eta_{i j}^{(0)}\right)+g\left(\eta_{i j}^{(0)}\right) g^{\prime \prime}\left(\eta_{i j}^{(0)}\right)\right] \boldsymbol{z}_{i j}^{\prime} D \boldsymbol{z}_{i j} .
\end{aligned}
$$

Likewise, for the covariance function:

$$
\operatorname{Cov}\left(Y_{i j}, Y_{i k}\right) \approx \sigma_{i j k}\left[g\left(\eta_{i j}^{(0)}\right) g\left(\eta_{i k}^{(0)}\right)+\frac{1}{2} g\left(\eta_{i j}^{(0)}\right) g^{\prime \prime}\left(\eta_{i k}^{(0)}\right) \boldsymbol{z}_{i k}^{\prime} D \boldsymbol{z}_{i k}\right.
$$




$$
\begin{aligned}
& \left.+\frac{1}{2} g\left(\eta_{i k}^{(0)}\right) g^{\prime \prime}\left(\eta_{i j}^{(0)}\right) \boldsymbol{z}_{i j}^{\prime} D \boldsymbol{z}_{i j}+g^{\prime}\left(\eta_{i j}^{(0)}\right) g^{\prime}\left(\eta_{i k}^{(0)}\right) \boldsymbol{z}_{i j}^{\prime} D \boldsymbol{z}_{i k}\right] \\
& +\vartheta_{i j} \xi_{i k} g^{\prime}\left(\eta_{i j}^{(0)}\right) g^{\prime}\left(\eta_{i k}^{(0)}\right) \boldsymbol{z}_{i j}^{\prime} D \boldsymbol{z}_{i k} .
\end{aligned}
$$

Here, $\sigma_{i j k}$ is the covariance between $\theta_{i j}$ and $\theta_{i k}$. In case these effects are assumed to be independent, a large portion of (A.3) then cancels, with covariance induced solely by the effects $\boldsymbol{b}_{\boldsymbol{i}}$. In case all $\theta_{i j}$ are equal, $\sigma_{i j k} \equiv \sigma_{i j}$. Evidently, (A.2) and (A.3) lead to approximate expressions for the correlations, too. Of course, in situations where closed forms exist, these expressions need not be used.

Needless to say that the above approximations may or may not be accurate, depending on the context. Therefore, their use should be seen as poor man's choice, when no explicit forms are available. Fortunately, closed forms are available for the normal (Section 4.4), Poisson (Section 4.5), and Weibull (Section 4.8) cases.

\section{B Computations for the Poisson Case}

Let us derive the mean and variance expressions for the combined model in the Poisson case.

The mean of a component can easily be derived as follows:

$$
\begin{aligned}
E\left(Y_{i j}\right) & =E\left\{E\left[E\left(Y_{i j} \mid \theta_{i j}, \boldsymbol{b}_{\boldsymbol{i}}\right)\right]\right\} \\
& =E\left\{E\left[\theta_{i j} \exp \left(\boldsymbol{x}_{i j}^{\prime} \boldsymbol{\xi}+\boldsymbol{z}_{i j}^{\prime} \boldsymbol{b}_{\boldsymbol{i}}\right)\right]\right\} \\
& =E\left\{\phi_{i j} \exp \left(\boldsymbol{x}_{i j}^{\prime} \boldsymbol{\xi}+\boldsymbol{z}_{i j}^{\prime} \boldsymbol{b}_{\boldsymbol{i}}\right)\right\} \\
& =\phi_{i j} \exp \left(\boldsymbol{x}_{i j}^{\prime} \boldsymbol{\xi}\right) E\left[\exp \left(\boldsymbol{z}_{i j}^{\prime} \boldsymbol{b}_{\boldsymbol{i}}\right)\right] \\
E\left(Y_{i j}\right) & =\phi_{i j} \exp \left(\boldsymbol{x}_{i j}^{\prime} \boldsymbol{\xi}+\frac{1}{2} \boldsymbol{z}_{i j}^{\prime} D \boldsymbol{z}_{i j}\right),
\end{aligned}
$$

where the last step follows from the following consideration. Note that the function $f=f_{i j}=\boldsymbol{z}_{i j}^{\prime} \boldsymbol{b}_{\boldsymbol{i}}$ is normally distributed with variance $d=\boldsymbol{z}_{i j}^{\prime} D \boldsymbol{z}_{i j}$. Hence, the expectation takes the form

$$
\begin{aligned}
E\left(e^{f}\right) & =\frac{1}{\sqrt{2 \pi} \sqrt{d}} \int e^{f} e^{-\frac{1}{2} \cdot \frac{f^{2}}{d}} d f \\
& =\frac{1}{\sqrt{2 \pi} \sqrt{d}} \int e^{-\frac{1}{2} \cdot \frac{(f-d)^{2}}{d}} e^{\frac{1}{2} d} d f \\
& =e^{\frac{1}{2} d} .
\end{aligned}
$$

Turning to the variance, a general expression is:

$$
\begin{aligned}
\operatorname{Var}\left(Y_{i j}\right) & =E\left\{E\left[\operatorname{Var}\left(Y_{i j} \mid \theta_{i j} \boldsymbol{b}_{\boldsymbol{i}}\right)\right]\right\}+E\left\{\operatorname{Var}\left[E\left(Y_{i j} \mid \theta_{i j} \boldsymbol{b}_{\boldsymbol{i}}\right)\right]\right\}+\operatorname{Var}\left\{E\left[E\left(Y_{i j} \mid \theta_{i j} \boldsymbol{b}_{\boldsymbol{i}}\right)\right]\right\} \\
& =T_{1}+T_{2}+T_{3} .
\end{aligned}
$$

We will derive expressions for each of the three terms in turn. Write $\theta_{i j} \exp \left(\boldsymbol{x}_{i j}^{\prime} \boldsymbol{\xi}+\boldsymbol{z}_{i j}^{\prime}\right)=\theta_{i j} \kappa_{i j}$ for brevity. Then it is easy to see, using that mean and variance are the same in the Poisson model, and equal to $\theta_{i j} \kappa_{i j}$ using our notation:

$$
\begin{aligned}
& T_{1}=E\left(\theta_{i j}\right) E\left(\kappa_{i j}\right), \\
& T_{2}=E\left(\theta_{i j}^{2}\right) E\left(\kappa_{i j}^{2}\right)-E\left(\theta_{i j}\right)^{2} E\left(\kappa_{i j}\right)^{2}, \\
& T_{3}=E\left(\theta_{i j}\right)^{2}\left[E\left(\kappa_{i j}^{2}\right)-E\left(\kappa_{i j}\right)^{2}\right],
\end{aligned}
$$


where expectations are taken over the appropriate random effect. Substituting (B.5)-(B.7) into (B.4) and simplifying produces:

$$
\operatorname{Var}\left(Y_{i j}\right)=E\left(\theta_{i j}\right) E\left(\kappa_{i j}\right)+E\left(\theta_{i j}^{2}\right) E\left(\kappa_{i j}^{2}\right)-E\left(\theta_{i j}\right)^{2} E\left(\kappa_{i j}\right)^{2} .
$$

Similar logic, applied to the covariance produces, for $j \neq k$ :

$$
\begin{aligned}
\operatorname{Cov}\left(Y_{i j}, Y_{i k}\right) & =E\left(\kappa_{i j} \kappa_{i k}\right) \operatorname{Cov}\left(\theta_{i j}, \theta_{i k}\right)+\operatorname{Cov}\left(\kappa_{i j}, \kappa_{i k}\right) E\left(\theta_{i j}\right) E\left(\theta_{i k}\right) \\
& =E\left(\kappa_{i j} \kappa_{i k}\right) E\left(\theta_{i j} \theta_{i k}\right)-E\left(\kappa_{i j}\right) E\left(\kappa_{i k}\right) E\left(\theta_{i j}\right) E\left(\theta_{i k}\right)
\end{aligned}
$$

Clearly, means, variances, and covariances of the $\theta_{i j}$ effects are

$$
E\left(\theta_{i j}\right)=\phi_{i j}, \quad \operatorname{Var}\left(\theta_{i j}\right)=\sigma_{i, j j}, \quad \operatorname{Cov}\left(\theta_{i j}, \theta_{i k}\right)=\sigma_{i, j k},
$$

respectively. For the $\kappa_{i j}$ components, (B.2) produces the mean form

$$
E\left(\kappa_{i j}\right)=e^{\boldsymbol{x}_{i j}^{\prime} \boldsymbol{\xi}+\frac{1}{2} \boldsymbol{z}_{i j}^{\prime} D \boldsymbol{z}_{i j}}
$$

Computations similar to (B.2) lead to:

$$
\operatorname{Var}\left(\kappa_{i j}\right)=e^{2 \boldsymbol{x}_{i j}^{\prime} \boldsymbol{\xi}+\boldsymbol{z}_{i j}^{\prime} D \boldsymbol{z}_{i j}} \cdot\left(e^{\boldsymbol{z}_{i j}^{\prime} D \boldsymbol{z}_{i j}}-1\right) .
$$

The corresponding covariance form is:

$$
\operatorname{Cov}\left(\kappa_{i j}, \kappa_{i k}\right)=e^{\boldsymbol{x}_{i j}^{\prime} \boldsymbol{\xi}+\boldsymbol{x}_{i k}^{\prime} \boldsymbol{\xi}+\frac{1}{2}\left(\boldsymbol{z}_{i j}^{\prime} D \boldsymbol{z}_{i j}+\boldsymbol{z}_{i k}^{\prime} D \boldsymbol{z}_{i k}\right)} \cdot\left(e^{\boldsymbol{z}_{i j}^{\prime} D \boldsymbol{z}_{i k}}-1\right) .
$$

Plugging (B.11)-(B.14) into (B.8) and (B.10), and re-organizing terms, leads to

$$
\operatorname{Var}\left(\boldsymbol{Y}_{\boldsymbol{i}}\right)=M_{i}+M_{i}\left(P_{i}-J_{n_{i}}\right) M_{i},
$$

where $M_{i}$ is defined in Section 3.2 and the $(j, k)^{\text {th }}$ element of $P_{i}$ equals

$$
p_{i, j k}=\exp \left(\frac{1}{2} \boldsymbol{z}_{i j}^{\prime} D z_{i k}\right) \cdot \frac{\sigma_{i, j k}+\phi_{i j} \phi_{i k}}{\phi_{i j} \phi_{i k}} \cdot \exp \left(\frac{1}{2} z_{i k}^{\prime} D \boldsymbol{z}_{i j}\right) .
$$

Clearly, these expressions also produce their simplified counterparts for the special cases, including the negative-binomial model, the Poisson-normal model, and the Poisson model, not only for the repeated-measures case, but clearly also for the further simplifications produced by considering univariate data only.

When only normal random effects are present, the above expressions simplify. The mean vector $\boldsymbol{\mu}_{i}=E\left(\boldsymbol{Y}_{\boldsymbol{i}}\right)$ slightly simplifies:

$$
\mu_{i j}=\exp \left(\boldsymbol{x}_{i j}^{\prime} \boldsymbol{\xi}+\frac{1}{2} \boldsymbol{z}_{i j}^{\prime} D \boldsymbol{z}_{i j}\right)
$$

with the variance-covariance matrix

$$
\operatorname{Var}\left(\boldsymbol{Y}_{\boldsymbol{i}}\right)=M_{i}+M_{i}\left(e^{Z_{i} D Z_{i}^{\prime}}-J_{n_{i}}\right) M_{i} .
$$


For information, if the variances and covariances are calculated from the general expression (A.2) and (A.3), then we find

$$
\begin{aligned}
\operatorname{Var}\left(Y_{i j}\right) & =E\left(\theta_{i j}\right) E\left(\kappa_{i j}\right)+E\left(\kappa_{i j}^{2}\right) E\left(\theta_{i j}^{2}\right)-E\left(\kappa_{i j}\right)^{2} E\left(\theta_{i j}\right)^{2}, \\
\operatorname{Cov}\left(Y_{i j}, Y_{i k}\right) & =E\left(\kappa_{i j} \kappa_{i k}\right) E\left(\theta_{i j} \theta_{i k}\right)-E\left(\kappa_{i j}\right) E\left(\kappa_{i k}\right) E\left(\theta_{i j}\right) E\left(\theta_{i k}\right) .
\end{aligned}
$$

As shown in Molenberghs, Verbeke, and Demétrio (2007), the joint probability of $\boldsymbol{Y}_{\boldsymbol{i}}$ can be derived if we additionally assume that the random effects follow Gamma distributions $\theta_{i j} \sim \operatorname{Gamma}\left(\alpha_{j}, \beta_{j}\right)$.

Because the Poisson-normal case follows from the combined model by taking limits over the gamma distribution only, it is easier not to follow this route but rather consider the Poisson-normal model separately. Let us do this first. To derive the marginal density, first write the terms of the linear predictor as $\boldsymbol{x}_{i j}^{\prime} \boldsymbol{\xi}=\gamma_{i j}$ and $\boldsymbol{z}_{i j}^{\prime} \boldsymbol{b}_{\boldsymbol{i}}=c_{i j}$, for convenience. The joint probability follows from:

$$
P\left(\boldsymbol{Y}_{\boldsymbol{i}}=\boldsymbol{y}_{\boldsymbol{i}}\right)=\int \prod_{i=1}^{n_{i}} \frac{1}{y_{i j} !}\left(e^{\gamma_{i j}+c_{i j}}\right)^{y_{i j}} e^{-e^{\gamma_{i j}+c_{i j}}} \frac{1}{|D|^{1 / 2}(2 \pi)^{q / 2}} e^{-\frac{1}{2} \boldsymbol{b}_{\boldsymbol{i}}{ }^{\prime} D \boldsymbol{b}_{\boldsymbol{i}}} d \boldsymbol{b}_{\boldsymbol{i}}
$$

We will use the following series expansion of the double exponential factor:

$$
e^{-e^{\gamma_{i j}+c_{i j}}}=\sum_{t=0}^{+\infty} \frac{(-1)^{t} e^{t \gamma_{i j}}}{t !} e^{t c_{i j}}
$$

Plugging this expression into (B.21) and re-ordering terms produces:

$$
\begin{aligned}
P\left(\boldsymbol{Y}_{\boldsymbol{i}}=\boldsymbol{y}_{\boldsymbol{i}}\right)= & \prod_{j=1}^{n_{i}} \frac{1}{y_{i j} !} \sum_{\boldsymbol{t}=\left(t_{1}, \ldots, t_{n_{i}}\right)} \frac{e^{\sum_{j=1}^{n_{i}}\left(y_{i j}+t_{j}\right) \gamma_{i j}}}{\prod_{j=1}^{n_{i}} \frac{1}{t_{j} !}}(-1)^{\sum_{j=1}^{n_{i}} t_{j}} \\
& \times \int e^{\sum_{j=1}^{n_{i}}\left(y_{i j}+t_{j}\right) c_{i j}} \frac{1}{|D|^{1 / 2}(2 \pi)^{q / 2}} e^{-\frac{1}{2} \boldsymbol{b}_{\boldsymbol{i}}{ }^{\prime} D \boldsymbol{b}_{\boldsymbol{i}}} d \boldsymbol{b}_{\boldsymbol{i}} .
\end{aligned}
$$

The integral in (B.22) can be reorganized in a fashion similar to (B.2), and hence it reduces to $\exp \left(0.5 \boldsymbol{w}_{i j}^{\prime} D \boldsymbol{w}_{i j}\right)$, with $\boldsymbol{w}_{i j}^{\prime}=\sum_{j=1}^{n_{i}}\left(y_{i j}+t_{j}\right) \boldsymbol{z}_{i j}^{\prime}$. Substituting this back in (B.22), and re-ordering factors, leads to:

$$
\begin{aligned}
P\left(\boldsymbol{Y}_{\boldsymbol{i}}=\boldsymbol{y}_{\boldsymbol{i}}\right)=\frac{1}{\prod_{j=1}^{n_{i}} y_{i j} !} \sum_{\boldsymbol{t}} & \frac{(-1)^{\sum_{j=1}^{n_{i}} t_{j}}}{\prod_{j=1}^{n_{i}} t_{j} !} \cdot \exp \left[\sum_{j=1}^{n_{i}}\left(y_{i j}+t_{j}\right) \boldsymbol{x}_{i j}^{\prime} \boldsymbol{\xi}\right] \\
& \times \exp \left\{\frac{1}{2}\left[\sum_{j=1}^{n_{i}}\left(y_{i j}+t_{j}\right) \boldsymbol{z}_{i j}^{\prime}\right] D\left[\sum_{j=1}^{n_{i}}\left(y_{i j}+t_{j}\right) \boldsymbol{z}_{i j}\right]\right\} .
\end{aligned}
$$

Now turning to the combined model, the parameters $\lambda_{i j}$ change to

$$
\lambda_{i j}=\theta_{i j} e^{\boldsymbol{x}_{i j}^{\prime} \boldsymbol{\xi}_{+} \boldsymbol{z}_{i j}^{\prime} \boldsymbol{b}_{\boldsymbol{i}}}=\theta_{i j} e^{\gamma_{i j}+c_{i j}}=e^{\widetilde{\gamma}_{i j}+c_{i j}},
$$

with $\gamma_{i j}$ and $c_{i j}$ the shorthand notation introduced above, and $\widetilde{\gamma}_{i j}$ absorbing the gamma random effect. With this notation, $P\left(\boldsymbol{Y}_{\boldsymbol{i}}=\boldsymbol{y}_{\boldsymbol{i}} \mid \boldsymbol{\eta}_{i}\right)$ takes the form (B.23). We then merely have to integrate 
further over the gamma random effect, assuming that $\theta_{i j}$ are independent, gamma distributed random variables $\theta_{i j} \sim \operatorname{Gamma}\left(\alpha_{j}, \beta_{j}\right)$. Re-expressing $\widetilde{\gamma}_{i j}$ in its constituents, the integral factor is:

$$
\frac{1}{\beta_{j}^{\alpha_{j}}} \frac{1}{\Gamma\left(\alpha_{j}\right)} \int \theta_{i j}^{\alpha_{j}-1} e^{-\theta_{i j} / \beta_{j}} \theta_{i j}^{y_{i j}+t_{j}} d \theta_{i j}=\frac{1}{\beta_{j}^{\alpha_{j}}} \frac{1}{\Gamma\left(\alpha_{j}\right)} \beta_{j}^{\alpha_{j}+y_{i j}+t_{j}} \Gamma\left(\alpha_{j}+y_{i j}+t_{j}\right) .
$$

Combining this expression with the other factors, re-ordering terms, and using properties of the Gamma function, produces

$$
\begin{aligned}
& P\left(\boldsymbol{Y}_{\boldsymbol{i}}=\boldsymbol{y}_{\boldsymbol{i}}\right)=\sum_{\boldsymbol{t}}\left[\prod_{j=1}^{n_{i}}\left(\begin{array}{c}
y_{i j}+t_{j} \\
y_{i j}
\end{array}\right) \cdot\left(\begin{array}{c}
\alpha_{j}+y_{i j}+t_{j}-1 \\
\alpha_{j}-1
\end{array}\right) \cdot(-1)^{t_{j}} \cdot \beta_{j}^{y_{i j}+t_{j}}\right] \\
& \times \exp \left(\sum_{j=1}^{n_{i}}\left(y_{i j}+t_{j}\right) \boldsymbol{x}_{i j}^{\prime} \boldsymbol{\xi}\right) \times \exp \left\{\frac{1}{2}\left[\sum_{j=1}^{n_{i}}\left(y_{i j}+t_{j}\right) \boldsymbol{z}_{i j}^{\prime}\right] D\left[\sum_{j=1}^{n_{i}}\left(y_{i j}+t_{j}\right) \boldsymbol{z}_{i j}\right]\right\} .
\end{aligned}
$$

In the above equation, the vector-valued index $\boldsymbol{t}=\left(t_{1}, \ldots, t_{n_{i}}\right)$ ranges over all non-negative integer vectors.

Obviously, the above computations simplify without problem to the special cases of the ordinary Poisson model and the negative-binomial models, respectively. The Poisson-normal and combined model expressions for univariate data follow without any problem, too.

\section{Computations for the Binary Case With Logit Link}

If, like in the Poisson case, the means, variances, and covariances are calculated from the general expression (A.2) and (A.3), then we find,

$$
\begin{aligned}
E\left(Y_{i j}\right) & =E\left(\theta_{i j}\right) E\left(\kappa_{i j}\right), \\
\operatorname{Var}\left(Y_{i j}\right) & =E\left(\theta_{i j}\right) E\left(\kappa_{i j}\right)-E\left(\kappa_{i j}\right)^{2} E\left(\theta_{i j}\right)^{2}, \\
\operatorname{Cov}\left(Y_{i j}, Y_{i k}\right) & =E\left(\kappa_{i j} . \kappa_{i k}\right) \cdot \operatorname{Cov}\left(\theta_{i j}, \theta_{i k}\right)+E\left(\theta_{i j}\right) E\left(\theta_{i k}\right) \cdot \operatorname{Cov}\left(\kappa_{i j}, \kappa_{i k}\right) .
\end{aligned}
$$

Note that, for the covariance, expression (B.20) and (C.3) are identical. Observe the difference between the Poisson and binary variance expressions, (B.19) and (C.2), respectively, underscoring that binary data cannot exhibit overdispersion except through correlation; this then also applies to binomial data.

Let us further consider (C.1)-(C.3) for the three situations with (1) the $\theta_{i j}$ generally correlated, (2) uncorrelated, and (3) equal to each other.

In the first case, we obtain:

$$
\begin{aligned}
E\left(Y_{i j}\right) & =\phi_{i j} E\left(\kappa_{i j}\right), \\
\operatorname{Var}\left(Y_{i j}\right) & =\phi_{i j} E\left(\kappa_{i j}\right)-\phi_{i j}^{2} E\left(\kappa_{i j}\right)^{2}, \\
\operatorname{Cov}\left(Y_{i j}, Y_{i k}\right) & =\sigma_{i j k} E\left(\kappa_{i j} . \kappa_{i k}\right)+\phi_{i j} \phi_{i k} \operatorname{Cov}\left(\kappa_{i j}, \kappa_{i k}\right) .
\end{aligned}
$$

In the second case, (C.4) and (C.5) remain the same, but

$$
\operatorname{Cov}\left(Y_{i j}, Y_{i k}\right)=\phi_{i j} \phi_{i k} \operatorname{Cov}\left(\kappa_{i j}, \kappa_{i k}\right) .
$$


In the third case, we obtain:

$$
\begin{aligned}
E\left(Y_{i j}\right) & =\phi_{i} E\left(\kappa_{i j}\right), \\
\operatorname{Var}\left(Y_{i j}\right) & =\phi_{i} E\left(\kappa_{i j}\right)-\phi_{i}^{2} E\left(\kappa_{i j}\right)^{2}, \\
\operatorname{Cov}\left(Y_{i j}, Y_{i k}\right) & =\sigma_{i}^{2} E\left(\kappa_{i j} . \kappa_{i k}\right)+\phi_{i}^{2} \operatorname{Cov}\left(\kappa_{i j}, \kappa_{i k}\right) .
\end{aligned}
$$

\section{Computations for the Binary Case With Probit Link}

We will sketch the derivation of (35). To this end, it is sufficient to integrate the joint density of $\boldsymbol{y}_{i}$ and $\boldsymbol{b}_{\boldsymbol{i}}$ over the random effect. It is evident how the beta part follows as a separate factor.

Because the components of $\boldsymbol{y}_{i}$ are independently, univariate normally distributed, given the random effects, we have for the marginal probability:

$$
\begin{aligned}
f & \left(\boldsymbol{y}_{i}=\mathbf{1}\right) \\
& =\left(\int_{-\infty}^{+\infty}\right)^{q} \int_{-\infty}^{X_{i}} \boldsymbol{\xi}_{+Z_{i} \boldsymbol{b}_{\boldsymbol{i}}} \frac{1}{(2 \pi)^{\left(n_{i}+q\right) / 2}|D|^{1 / 2}} \exp \left\{-\frac{1}{2}\left[\boldsymbol{b}_{\boldsymbol{i}}{ }^{\prime} D^{-1} \boldsymbol{b}_{\boldsymbol{i}}+\sum_{j=1}^{n_{i}} t_{j}^{2}\right]\right\} d \boldsymbol{b}_{\boldsymbol{i}} d \boldsymbol{t} \\
& =\left(\int_{-\infty}^{+\infty}\right)^{q} \int_{-\infty}^{X_{i} \boldsymbol{\xi}} \frac{1}{(2 \pi)^{\left(n_{i}+q\right) / 2}|D|^{1 / 2}} \exp \left\{-\frac{1}{2}\left[\boldsymbol{b}_{\boldsymbol{i}}{ }^{\prime} D^{-1} \boldsymbol{b}_{\boldsymbol{i}}+\left(\boldsymbol{s}+Z_{i} \boldsymbol{b}_{\boldsymbol{i}}\right)^{\prime}\left(\boldsymbol{s}+Z_{i} \boldsymbol{b}_{\boldsymbol{i}}\right)\right]\right\} d \boldsymbol{b}_{\boldsymbol{i}} d \boldsymbol{s} \\
= & \left(\int_{-\infty}^{+\infty}\right)^{q} \int_{-\infty}^{X_{i} \boldsymbol{\xi}} \frac{1}{(2 \pi)^{\left(n_{i}+q\right) / 2}|D|^{1 / 2} \cdot|L|^{1 / 2} \cdot|K|^{1 / 2} \cdot|K|^{-1 / 2} \cdot|L|^{-1 / 2}} \\
= & \times \frac{1}{|D|^{1 / 2} \cdot|L|^{1 / 2} \cdot|K|^{1 / 2}} \cdot \Phi_{n_{i}}\left(X_{i} \boldsymbol{\xi}, L^{-1}\right) \\
= & \Phi_{n_{i}}\left(X_{i} \boldsymbol{\xi}, L^{-1}\right) .
\end{aligned}
$$

Here, we have made the change of variable $\boldsymbol{t}=\boldsymbol{s}+Z_{i} \boldsymbol{b}_{\boldsymbol{i}}$, and further

$$
\boldsymbol{b}_{\boldsymbol{i}}{ }^{\prime} D^{-1} \boldsymbol{b}_{\boldsymbol{i}}+\left(\boldsymbol{s}+Z_{i} \boldsymbol{b}_{\boldsymbol{i}}\right)^{\prime}\left(\boldsymbol{s}+Z_{i} \boldsymbol{b}_{\boldsymbol{i}}\right)=\boldsymbol{b}_{\boldsymbol{i}}{ }^{\prime} K \boldsymbol{b}_{\boldsymbol{i}}+2 \boldsymbol{s}^{\prime} Z_{i} \boldsymbol{b}_{\boldsymbol{i}}+\boldsymbol{s}^{\prime} \boldsymbol{s}=\left(\boldsymbol{b}_{\boldsymbol{i}}-\boldsymbol{k}\right)^{\prime} K\left(\boldsymbol{b}_{\boldsymbol{i}}-\boldsymbol{k}\right)+\ell,
$$

where

$$
\begin{aligned}
K & =D^{-1}+Z_{i}^{\prime} Z_{i}, \\
\boldsymbol{k} & =-K^{-1} Z_{i}^{\prime} \boldsymbol{s}, \\
\ell & =\boldsymbol{s}^{\prime} L \boldsymbol{s}, \\
L & =I_{n_{i}}-Z_{i} K^{-1} Z_{i}^{\prime} .
\end{aligned}
$$

To establish the transition from (D.1) tot (D.2), write

$$
A=\left(\begin{array}{c|c}
K & Z_{i}^{\prime} \\
\hline Z_{i} & I
\end{array}\right) .
$$


Based on the determinant equality for patterned matrices:

$$
\begin{aligned}
|A|=|K| \cdot\left|I-Z_{i} K^{-1} Z_{i}^{\prime}\right| & =|I| \cdot\left|K-Z_{i}^{\prime} I Z_{i}\right| \\
|K| \cdot|L| & =\left|D^{-1}+Z_{i}^{\prime} Z_{i}-Z_{i}^{\prime} Z_{i}\right| \\
|K| \cdot|L| \cdot|D| & =1 .
\end{aligned}
$$

Note that (35), strictly speaking, the joint probability only for the outcome $(1, \ldots, 1)^{\prime}$, a so-called success probability. However, given that the dimension $n_{i}$ is arbitrary, all other probabilities can be derived by appropriate contrasts of success probabilities. Precisely,

$$
f_{n_{i}}\left[\boldsymbol{y}_{i}=\boldsymbol{m}_{i}=\left(m_{i 1}, \ldots, m_{i n_{i}}\right)^{\prime}\right]=\sum_{\boldsymbol{s} \supset \lambda\left(\boldsymbol{m}_{i}\right)} \operatorname{sgn}(\boldsymbol{s}) \Phi_{\# \boldsymbol{s}}\left(\widetilde{X}_{i}^{(\boldsymbol{s})} \boldsymbol{\xi} ; L_{(\boldsymbol{s})}^{-1}\right) \cdot\left(\frac{\alpha}{\alpha+\beta}\right)^{\# \boldsymbol{s}},
$$

with $\lambda\left(\boldsymbol{m}_{i}\right)=\lambda\left(m_{i 1}, \ldots, m_{i n_{i}}\right)$ the set of places for which $m_{i j}=1$,

$$
\operatorname{sgn}(s)= \begin{cases}1 & \text { if } \# \boldsymbol{s}-\# \lambda\left(\boldsymbol{m}_{i}\right) \text { is even } \\ 0 & \text { otherwise }\end{cases}
$$

$\widetilde{X}_{i}^{(\boldsymbol{s})}$ contains the rows from $X_{i}$ with row number in $s$, and $L_{(s)}$ is the \#s-dimensional matrix built from the appropriate sub-matrices of these used in (36). The above developments straightforwardly generalize when (34) is replaced with $\theta_{i j} \sim \operatorname{Beta}\left(\alpha_{j}, \beta_{j}\right)$.

Next, the means, variances, and covariances can be derived from (35), by evaluating it for the oneand two-dimensional cases. We find:

$$
\begin{aligned}
E\left(Y_{i j}\right) & =\frac{\alpha}{\alpha+\beta} \cdot \Phi_{1}\left(\boldsymbol{x}_{i j}^{\prime} \boldsymbol{\xi} ; L_{1}^{-1}\right)=\frac{\alpha}{\alpha+\beta} \cdot \Phi_{1}\left(\left|I+D z_{i j} z_{i j}^{\prime}\right|^{-1 / 2} \boldsymbol{x}_{i j}^{\prime} \boldsymbol{\xi}\right) \\
\operatorname{Var}\left(Y_{i j}\right) & =\frac{\alpha}{\alpha+\beta} \cdot \Phi_{1}\left(\boldsymbol{x}_{i j}^{\prime} \boldsymbol{\xi} ; L_{1}^{-1}\right) \cdot\left[1-\frac{\alpha}{\alpha+\beta} \cdot \Phi_{1}\left(\boldsymbol{x}_{i j}^{\prime} \boldsymbol{\xi} ; L_{1}^{-1}\right)\right] \\
\operatorname{Cov}\left(Y_{i j}, Y_{i k}\right) & =\left(\frac{\alpha}{\alpha+\beta}\right)^{2} \cdot\left\{\Phi_{2}\left[\left(\begin{array}{c}
\boldsymbol{x}_{i j}^{\prime} \\
\boldsymbol{x}_{i k}^{\prime}
\end{array}\right) \boldsymbol{\xi}, L_{2 j k}^{-1}\right]-\Phi_{1}\left(\boldsymbol{x}_{i j}^{\prime} \boldsymbol{\xi} ; L_{1 j}^{-1}\right) \Phi_{1}\left(\boldsymbol{x}_{i k}^{\prime} \boldsymbol{\xi} ; L_{1 k}^{-1}\right)\right\}(, \mathrm{D} .0
\end{aligned}
$$

where

$$
L_{2 j k}=I_{2}-\left(\begin{array}{c}
\boldsymbol{z}_{i j}^{\prime} \\
\boldsymbol{z}_{i k}^{\prime}
\end{array}\right)\left[D^{-1}+\left(\begin{array}{c}
\boldsymbol{z}_{i j}^{\prime} \\
\boldsymbol{z}_{i k}^{\prime}
\end{array}\right)\left(\boldsymbol{z}_{i j} \boldsymbol{z}_{i k}\right)\right]^{-1}\left(\boldsymbol{z}_{i j} \boldsymbol{z}_{i k}\right) .
$$

Note that the rightmost density in (D.4) is the standard normal one. Evidently, (35) and (D.3) lead, not only to the mean, variance, and covariance expressions, but also to the higher-order moments.

\section{E Computations for the Time-to-event Case}

Let us derive the marginal density of the model specified by (41)-(42). First replace the predictor $\boldsymbol{x}_{i j}^{\prime} \boldsymbol{\xi}+\boldsymbol{z}_{i j}^{\prime} \boldsymbol{b}_{\boldsymbol{i}}$ in (41) by $\mu$, and integrate

$$
f(y \mid \theta)=\lambda \rho y^{\rho-1} \theta e^{\mu} e^{-\lambda y^{\rho} \theta e^{\mu}}
$$


over the general gamma distribution of $\theta$, i.e., over one component of (42):

$$
f(y)=\frac{\lambda \rho y^{\rho-1} e^{\mu}}{\beta^{\alpha} \Gamma(\alpha)} \int \theta^{\alpha} e^{-\theta\left[1 / \beta+\lambda y^{\rho} e^{\mu}\right]} d \theta=\frac{\lambda \rho y^{\rho-1} e^{\mu} \alpha \beta}{\left(1+\lambda \beta y^{\rho} e^{\mu}\right)^{\alpha+1}},
$$

which easily follows upon setting $z=1 / \beta+\lambda y^{\rho} e^{\mu}$.

Now, for the general case, first observe that

$$
e^{-\lambda y_{i j}^{\rho} \theta_{i j} e^{\mu_{i j}+\boldsymbol{z}_{i j}^{\prime} \boldsymbol{b}_{\boldsymbol{i}}}}=\sum_{m_{j}=0}^{+\infty} \frac{(-1)^{m_{j}}}{m_{j} !} \lambda^{m_{j}} y_{i j}^{m_{j} \rho} \theta_{i j}^{m_{j}} e^{m_{j}\left(\mu_{i j}+\boldsymbol{z}_{i j}^{\prime} \boldsymbol{b}_{\boldsymbol{i}}\right)} .
$$

It then follows that

$$
\begin{aligned}
& f\left(\boldsymbol{y}_{i} \mid \boldsymbol{\theta}_{i}\right)=\prod_{j=1}^{n_{i}} \lambda \rho \theta_{i j} y_{i j}^{\rho-1} \\
& \times \frac{1}{(2 \pi)^{q / 2}|D|^{1 / 2}} \int e^{\mu_{i j}+\boldsymbol{z}_{i j}^{\prime} \boldsymbol{b}_{\boldsymbol{i}}} e^{-\lambda y_{i j}^{\rho} \theta_{i j} e^{\mu_{i j}+\boldsymbol{z}_{i j}^{\prime} \boldsymbol{b}_{\boldsymbol{i}}}} e^{-\frac{1}{2} \boldsymbol{b}_{\boldsymbol{i}}{ }^{\prime} D^{-1} \boldsymbol{b}_{\boldsymbol{i}}} d \boldsymbol{b}_{\boldsymbol{i}} \\
& =\sum_{\left(m_{1}, \ldots, m_{n_{i}}\right)} \prod_{j=1}^{n_{i}} \frac{(-1)^{m_{j}}}{m_{j} !} \lambda^{m_{j}+1} \rho y_{i j}^{\left(m_{j}+1\right) \rho-1} \theta_{i j}^{m_{j}+1} \\
& \times \frac{1}{(2 \pi)^{q / 2}|D|^{1 / 2}} \int e^{\left(m_{j}+1\right)\left(\mu_{i j}+\boldsymbol{z}_{i j}^{\prime} \boldsymbol{b}_{\boldsymbol{i}}\right)-\frac{1}{2} \boldsymbol{b}_{\boldsymbol{i}}{ }^{\prime} D^{-1} \boldsymbol{b}_{\boldsymbol{i}}} d \boldsymbol{b}_{\boldsymbol{i}}
\end{aligned}
$$

Now, similar to the binary case in Appendix $C$, write

$$
-\frac{1}{2} \boldsymbol{b}_{\boldsymbol{i}}{ }^{\prime} D^{-1} \boldsymbol{b}_{\boldsymbol{i}}+\left(m_{j}+1\right)\left(\mu_{i j}+\boldsymbol{z}_{i j}^{\prime} \boldsymbol{b}_{\boldsymbol{i}}\right)=-\frac{1}{2}\left(\boldsymbol{b}_{\boldsymbol{i}}-\boldsymbol{t}\right)^{\prime} D^{-1}\left(\boldsymbol{b}_{\boldsymbol{i}}-\boldsymbol{t}\right)+\ell,
$$

with

$$
\boldsymbol{t}=\left(m_{j}+1\right) D \boldsymbol{z}_{i j}, \quad \ell=\left(m_{j}+1\right)\left[\mu_{i j}+\frac{1}{2}\left(m_{j}+1\right) \boldsymbol{z}_{i j}^{\prime} D \boldsymbol{z}_{i j}\right] .
$$

Combining (E.3) with (E.4) produces

$$
f\left(\boldsymbol{y}_{i} \mid \boldsymbol{\theta}_{i}\right)=\sum_{\left(m_{1}, \ldots, m_{n_{i}}\right)} \prod_{j=1}^{n_{i}} \frac{(-1)^{m_{j}}}{m_{j} !} \lambda^{m_{j}+1} \rho y_{i j}^{\left(m_{j}+1\right) \rho-1} \theta_{i j}^{m_{j}+1} e^{\left(m_{j}+1\right)\left[\mu_{i j}+\frac{1}{2}\left(m_{j}+1\right) \boldsymbol{z}_{i j}^{\prime} D \boldsymbol{z}_{i j}\right]} .
$$

Further integration over the gamma distribution produces

$$
f\left(\boldsymbol{y}_{i}\right)=\sum_{\left(m_{1}, \ldots, m_{n_{i}}\right)} \prod_{j=1}^{n_{i}} \frac{(-1)^{m_{j}}}{m_{j} !} \frac{\lambda^{m_{j}+1} \rho y_{i j}^{\left(m_{j}+1\right) \rho-1} e^{\left(m_{j}+1\right)\left[\mu_{i j}+\frac{1}{2}\left(m_{j}+1\right) \boldsymbol{z}_{i j}^{\prime} D \boldsymbol{z}_{i j}\right]}}{\beta_{j}^{\alpha_{j}} \Gamma\left(\alpha_{j}\right)} \cdot I_{j, m_{j}}
$$

with

$$
I_{j, m_{j}}=\int \theta_{i j}^{m_{j}+\alpha_{j}} e^{-\theta_{i j} / \beta_{j}} d \theta_{i j}=\beta_{j}^{m_{j}+\alpha_{j}+1} \Gamma\left(m_{j}+\alpha_{j}+1\right) .
$$

Plugging (E.6) into (E.7) yields

$$
\begin{aligned}
f\left(\boldsymbol{y}_{i}\right)=\sum_{\left(m_{1}, \ldots, m_{n_{i}}\right)} \prod_{j=1}^{n_{i}} & \frac{(-1)^{m_{j}}}{m_{j} !} \frac{\Gamma\left(\alpha_{j}+m_{j}+1\right) \beta_{j}^{m_{j}+1}}{\Gamma\left(\alpha_{j}\right)} \lambda^{m_{j}+1} \rho y_{i j}^{\left(m_{j}+1\right) \rho-1} \\
& \times \exp \left\{\left(m_{j}+1\right)\left[\boldsymbol{x}_{i j}^{\prime} \boldsymbol{\xi}+\frac{1}{2}\left(m_{j}+1\right) \cdot \boldsymbol{z}_{i j}^{\prime} D \boldsymbol{z}_{i j}\right]\right\} .
\end{aligned}
$$


As for the moments, let us first derive the moments based upon (E.1):

$$
\begin{aligned}
E\left(Y^{k}\right) & =\int_{0}^{+\infty} \frac{\lambda \rho y^{\rho-1+k} e^{\mu} \alpha \beta}{\left(1+\lambda \beta y^{\rho} e^{\mu}\right)^{\alpha+1}} d y \\
& =\frac{\alpha}{\varphi^{k / \rho} \beta^{k / \rho}} \int_{1}^{+\infty} t^{-\alpha-1}(t-1)^{k / \rho} d t \\
& =\frac{\alpha}{\varphi^{k / \rho} \beta^{k / \rho}} \int_{0}^{1} z^{\alpha-1-k / \rho}(1-z)^{k / \rho} d z \\
& =\frac{\alpha}{\varphi^{k / \rho} \beta^{k / \rho}} B(\alpha-k / \rho, k / \rho+1),
\end{aligned}
$$

where $\varphi=\lambda e^{\mu}$, and the integrator transformations $t=1+\lambda \beta y^{\rho} e^{\mu}$ and $t=1 / z$ have been used. Now, (E.9) can be used as the $k$ th moment, conditional upon $\boldsymbol{b}_{\boldsymbol{i}}$, as follows:

$$
E\left(Y_{i j}^{k} \mid \boldsymbol{b}_{\boldsymbol{i}}\right)=\frac{\alpha_{j}}{\varphi_{i j}^{k / \rho} \beta_{j}^{k / \rho}} B\left(\alpha_{j}-k / \rho, k / \rho+1\right),
$$

where $\varphi_{i j}=\lambda e^{\mu_{i j}+\boldsymbol{z}_{i j}^{\prime} \boldsymbol{b}_{\boldsymbol{i}}}$. The unconditional moment follows as:

$$
\begin{aligned}
E\left(Y_{i j}^{k}\right) & =\frac{\alpha_{j} B\left(\alpha_{j}-k / \rho, k / \rho+1\right)}{\lambda^{k / \rho} e^{\mu_{i j} k / \rho} \beta_{j}^{k / \rho}} \frac{1}{(2 \pi)^{q / 2}|D|^{1 / 2}} \int e^{-\frac{1}{2} \boldsymbol{b}_{\boldsymbol{i}}{ }^{\prime} D^{-1} \boldsymbol{b}_{\boldsymbol{i}}-\frac{k}{\rho} \boldsymbol{z}_{i j}^{\prime} \boldsymbol{b}_{\boldsymbol{i}}} d \boldsymbol{b}_{\boldsymbol{i}} \\
& =\frac{\alpha_{j} B\left(\alpha_{j}-k / \rho, k / \rho+1\right)}{\lambda^{k / \rho} e^{\mu_{i j} k / \rho} \beta_{j}^{k / \rho}} e^{\frac{k^{2}}{2 \rho^{2}} \boldsymbol{z}_{i j}^{\prime} D \boldsymbol{z}_{i j}},
\end{aligned}
$$

where we rewrote

$$
-\frac{1}{2} \boldsymbol{b}_{\boldsymbol{i}}{ }^{\prime} D^{-1} \boldsymbol{b}_{\boldsymbol{i}}-\frac{k}{\rho} \boldsymbol{z}_{i j} \boldsymbol{b}_{\boldsymbol{i}}=-\frac{1}{2}\left(\boldsymbol{b}_{\boldsymbol{i}}-\boldsymbol{t}\right)^{\prime} D^{-1}\left(\boldsymbol{b}_{\boldsymbol{i}}-\boldsymbol{t}\right)+\ell,
$$

with

$$
\boldsymbol{t}=\frac{k}{\rho} D \boldsymbol{z}_{i j}, \quad \ell=\frac{1}{2} \frac{k^{2}}{\rho^{2}} \boldsymbol{z}_{i j}^{\prime} D \boldsymbol{z}_{i j} .
$$

From (E.11), we immediately derive, the following moment expression, with mean, variance, and covariance expressions:

$$
\begin{aligned}
E\left(Y_{i j}^{k}\right)= & \frac{\alpha_{j} B\left(\alpha_{j}-k / \rho, k / \rho+1\right)}{\lambda^{k / \rho} \beta_{j}^{k / \rho}} \exp \left(-\frac{k}{\rho} \boldsymbol{x}_{i j}^{\prime} \boldsymbol{\xi}+\frac{k^{2}}{2 \rho^{2}} \boldsymbol{z}_{i j}^{\prime} D \boldsymbol{z}_{i j}\right) \\
E\left(Y_{i j}\right)= & \frac{\alpha_{j} B\left(\alpha_{j}-1 / \rho, 1 / \rho+1\right)}{\lambda^{1 / \rho} \beta_{j}^{1 / \rho}} \exp \left(-\frac{1}{\rho} \boldsymbol{x}_{i j}^{\prime} \boldsymbol{\xi}+\frac{1}{2 \rho^{2}} \boldsymbol{z}_{i j}^{\prime} D \boldsymbol{z}_{i j}\right) \\
\operatorname{Var}\left(Y_{i j}\right)= & \frac{\alpha_{j}}{\lambda^{2 / \rho} \beta_{j}^{2 \rho}} \exp \left(-\frac{2}{\rho} \boldsymbol{x}_{i j}^{\prime} \boldsymbol{\xi}+\frac{1}{\rho^{2}} \boldsymbol{z}_{i j}^{\prime} D \boldsymbol{z}_{i j}\right) \\
& \times\left[B\left(\alpha_{j}-2 / \rho, 2 / \rho+1\right) \exp \left(\frac{1}{\rho^{2}} \boldsymbol{z}_{i j}^{\prime} D \boldsymbol{z}_{i j}\right)-\alpha_{j} B\left(\alpha_{j}-\frac{1}{\rho}, \frac{1}{\rho}+1\right)^{2}\right] \mathrm{E}_{,} \\
\operatorname{Cov}\left(Y_{i j}, Y_{i k}\right)= & \frac{\alpha_{j} \alpha_{k}}{\lambda^{2 / \rho} \beta_{j}^{1 / \rho} \beta_{k}^{1 / \rho}} \exp \left[-\frac{1}{\rho}\left(\boldsymbol{x}_{i j}^{\prime} \boldsymbol{\xi}+\boldsymbol{x}_{i k}^{\prime} \boldsymbol{\xi}\right)\right]
\end{aligned}
$$




$$
\begin{aligned}
& \times B\left(\alpha_{j}-\frac{1}{\rho}, \frac{1}{\rho}+1\right) B\left(\alpha_{k}-\frac{1}{\rho}, \frac{1}{\rho}+1\right) \\
& \times \exp \left[\frac{1}{2 \rho^{2}}\left(\boldsymbol{z}_{i j}^{\prime} D \boldsymbol{z}_{i j}+\boldsymbol{z}_{i k}^{\prime} D \boldsymbol{z}_{i k}\right)\right]\left[\exp \left(\frac{1}{\rho^{2}} \boldsymbol{z}_{i j}^{\prime} D \boldsymbol{z}_{i k}\right)-1\right] .
\end{aligned}
$$

It is customary, in the standard frailty model (Duchateau and Janssen 2007), to set $\beta_{j}=1 / \alpha_{j}$, as in (44) for identifiability purposes. The change to (E.8) on the one hand, and to (E.12)-(E.15) is then both evident and minor. Likewise, the exponential version follows from setting $\rho=1$.

It is of interest to explore in a bit more detail the special case induced by (45). Setting $\alpha_{j}=1$ and $\beta_{j}=1 / \delta_{j}$ implies, for (E.12), upon some rewriting:

$$
E\left(Y_{i j}^{k}\right)=\frac{\delta_{j}^{k / \rho} k}{\lambda^{k / \rho}} \Gamma\left(1-\frac{k}{\rho}\right) \Gamma\left(\frac{k}{\rho}\right) \exp \left(-\frac{k}{\rho} \boldsymbol{x}_{i j}^{\prime} \boldsymbol{\xi}+\frac{k^{2}}{2 \rho^{2}} \boldsymbol{z}_{i j}^{\prime} D \boldsymbol{z}_{i j}\right) .
$$

Reducing the Weibull distribution to the exponential one, i.e., setting $\rho=1$, we find:

$$
E\left(Y_{i j}^{k}\right)=\frac{\delta_{j}^{k} k}{\lambda^{k}} \Gamma(1-k) \Gamma(k) \exp \left(-k \boldsymbol{x}_{i j}^{\prime} \boldsymbol{\xi}+\frac{k^{2}}{2} \boldsymbol{z}_{i j}^{\prime} D \boldsymbol{z}_{i j}\right) .
$$

The cases corresponding to (E.16) and, especially, (E.17) deserve further attention. Generally, $\Gamma(\alpha-k / \rho)$ poses a problem when $\alpha-k / \rho$ is a negative integer. For simplicity focusing on a single outcome $Y$ for the case where $\alpha=1$ and $\beta=1 / \delta$ assuming there are no normal random effects, assembling the linear predictor in $\mu$, and writing $\varphi=\lambda e^{\mu}$, we find:

$$
\begin{aligned}
f(y) & =\frac{\varphi \rho y^{\rho-1} \delta}{\left(\delta+\varphi y^{\rho}\right)^{2}} \\
E\left(Y^{k}\right) & =\frac{k}{\rho}\left(\frac{\delta}{\varphi}\right)^{k / \rho} \cdot \Gamma(1-k / \rho) \cdot \Gamma(k / \rho)
\end{aligned}
$$

Note that (E.18) provides a family of distributions, special cases of the Weibull-gamma model in Table 1 that we could describe as Weibull-exponential. Considering further the exponential case with $\rho=1$, yields exponential-exponential distributions, with:

$$
\begin{aligned}
f(y) & =\frac{\varphi \delta}{(\delta+\varphi y)^{2}}, \\
E\left(Y^{k}\right) & =k\left(\frac{\delta}{\varphi}\right)^{k} \cdot \Gamma(1-k) \cdot \Gamma(k) .
\end{aligned}
$$

Clearly, (E.20) defines a family of distributions without finite moments, exactly like but different from the Cauchy distribution, because $\Gamma(1-k)$ is undefined for $k=1,2, \ldots$ When $\rho \neq 1$ but fractional, some but not all moments in (E.19) exist, whereas for irrational values of $\rho$, all moments in (E.19) are properly defined. Finally, observe that in the general case, there are combinations possible for $(\alpha, \rho, k)$ that would lead to negative integers and hence undefined moments (E.16).

Specification (E.18) opens the door for the construction of distributions with arbitrarily wide tails, yet finite moments. it suffices to choose $\rho=1+\epsilon$, with $\epsilon$ an arbitrarily small irrational value.

Still concentrating on the single-outcome case for notational simplicity, note that the mean-variance relationship, for the Weibull model with normal and gamma random effect, takes the form:

$$
\operatorname{Var}(Y)=E(Y)^{2}\left[\frac{2 B\left(\alpha-\frac{2}{\rho}, \frac{2}{\rho}\right)}{B\left(\alpha-\frac{1}{\rho}, \frac{1}{\rho}\right)^{2}} \cdot \rho \cdot e^{d / \rho^{2}}-1\right] .
$$


When $\rho=1$, this becomes

$$
\operatorname{Var}(Y)=E(Y)^{2}\left[\frac{2(\alpha-1)}{\alpha-2} e^{d}-1\right]
$$

Finally, when also $d=0$, we find:

$$
\operatorname{Var}(Y)=E(Y)^{2}\left(\frac{\alpha}{\alpha-2}\right)
$$

\section{F Marginal Correlation}

As we made clear in Section 4.9, a variety of quantities, derived from the marginal distribution and its moments are of interest and become within reach. Perhaps the most prominent one is the correlation function. Depending on the context, these can take the form of pairwise correlations, intraclass correlations, etc.

We will first discuss a Taylor-series-based approximation method, apply it to the Poisson and binary cases, and then switch to the closed-form expressions.

\section{F.1 Generic Approximations}

Vangeneugden et al (2008a) derived, and Vangeneugden et al (2008b) further used approximate expressions for the correlation function in the GLMM, including when multiple sequences on the same subject are observed. For situations where there are no closed-form expressions available, for the joint distributions and/or for the moments, this is pragmatically a sensible way forward. Even when closed forms are available, the derivation of approximate expressions is still useful, not only to evaluate the quality of approximations, but also to cater for situations where the closed forms would be too cumbersome.

We can usefully write the general model as $\boldsymbol{Y}_{i}=\boldsymbol{\mu}_{i}+\boldsymbol{\varepsilon}_{i}$, where $\boldsymbol{\mu}_{i}$, the conditional mean, given the random effects, can be written as $\boldsymbol{\mu}_{i}=h\left(\boldsymbol{X}_{i} \boldsymbol{\xi}+\boldsymbol{Z}_{i} \boldsymbol{b}_{i}\right), \boldsymbol{X}_{i}$ and $\boldsymbol{Z}_{i}$ are known design matrices, $\boldsymbol{\xi}$ are fixed-effect parameters, $\boldsymbol{b}_{i}$ are random effects, and $h$ is a known link function. Finally, $\boldsymbol{\varepsilon}_{i}$ is the residual error component. We will now derive a general formula for the variance-covariance matrix of $\boldsymbol{Y}_{i}$ without any restriction on the distribution of the outcome variable nor on the complexity of the model, e.g., allowing for serial correlation or not. This maximizes the similarity with the case of continuous, normally distributed outcomes. However, a key distinction is that in the linear case there is no mean-variance link, whereas here the residual variance will follow from the mean. The variance-covariance matrix can be written as:

$$
\boldsymbol{V}_{i}=\operatorname{Var}\left(\boldsymbol{Y}_{i}\right)=\operatorname{Var}\left(\boldsymbol{\mu}_{i}+\boldsymbol{\varepsilon}_{i}\right)=\operatorname{Var}\left(\boldsymbol{\mu}_{i}\right)+\operatorname{Var}\left(\boldsymbol{\varepsilon}_{i}\right)+2 \operatorname{Cov}\left(\boldsymbol{\mu}_{i}, \boldsymbol{\varepsilon}_{i}\right) .
$$

It is easy to show that $\operatorname{Cov}\left(\boldsymbol{\mu}_{i}, \boldsymbol{\varepsilon}_{i}\right)=\operatorname{Cov}\left[\mathrm{E}\left(\boldsymbol{\mu}_{i} \mid \boldsymbol{b}_{i}\right), \mathrm{E}\left(\varepsilon_{i} \mid \boldsymbol{b}_{i}\right)\right]+\mathrm{E}\left[\operatorname{Cov}\left(\boldsymbol{\mu}_{i}, \boldsymbol{\varepsilon}_{i} \mid \boldsymbol{b}_{i}\right)\right]=0$ since the first term is 0 and the second term equals $\mathrm{E}\left[\mathrm{E}\left(\boldsymbol{\mu}_{i}-\mathrm{E}\left(\boldsymbol{\mu}_{i}\right)\right)\left(\boldsymbol{\varepsilon}_{i}\right) \mid \boldsymbol{b}_{\boldsymbol{i}}\right]=0$ as $\boldsymbol{\mu}_{i}$ is a constant when conditioning on $\boldsymbol{b}_{i}$. For the first term in (F.1) we have, using a first-order Taylor series expansion around $\boldsymbol{b}_{i}=\mathbf{0}$ :

$$
\begin{aligned}
\operatorname{Var}\left(\boldsymbol{\mu}_{i}\right) & =\operatorname{Var}\left[\boldsymbol{\mu}_{i}\left(\boldsymbol{\eta}_{\boldsymbol{i}}\right)\right]=\operatorname{Var}\left[\boldsymbol{\mu}_{i}\left(\boldsymbol{X}_{i} \boldsymbol{\xi}+\boldsymbol{Z}_{i} \boldsymbol{b}_{i}\right)\right] \\
& \cong\left(\left.\frac{\partial \boldsymbol{\mu}_{i}}{\partial \boldsymbol{\eta}_{\boldsymbol{i}}} \frac{\partial \boldsymbol{\eta}_{\boldsymbol{i}}}{\partial \boldsymbol{b}_{i}}\right|_{\boldsymbol{b}_{i}=0}\right) \boldsymbol{D}\left(\left.\frac{\partial \boldsymbol{\mu}_{i}}{\partial \boldsymbol{\eta}_{\boldsymbol{i}}} \frac{\partial \boldsymbol{\eta}_{\boldsymbol{i}}}{\partial \boldsymbol{b}_{i}}\right|_{\boldsymbol{b}_{i}=0}\right)^{\prime} \cong \boldsymbol{\Delta}_{i} \boldsymbol{Z}_{i} \boldsymbol{D} \boldsymbol{Z}_{i}^{\prime} \boldsymbol{\Delta}_{i}^{\prime},
\end{aligned}
$$


where $\boldsymbol{\Delta}_{i}=\left.\frac{\partial \boldsymbol{\mu}_{i}}{\partial \boldsymbol{\eta}_{\boldsymbol{i}}}\right|_{\boldsymbol{b}_{i}=0}$. For the second term in (F.1), we have:

$$
\operatorname{Var}\left(\varepsilon_{i}\right)=\operatorname{Var}\left[E\left(\varepsilon_{i} \mid \boldsymbol{b}_{i}\right)\right]+E\left[\operatorname{Var}\left(\varepsilon_{i} \mid \boldsymbol{b}_{i}\right)\right]=E\left[\operatorname{Var}\left(\varepsilon_{i} \mid \boldsymbol{b}_{i}\right)\right]=\boldsymbol{\Phi}^{\frac{1}{2}} \boldsymbol{\Sigma}_{i} \boldsymbol{\Phi}^{\frac{1}{2}},
$$

where $\boldsymbol{\Phi}$ is a diagonal matrix with the overdispersion parameters along the diagonal. In case there are no overdispersion parameters, $\boldsymbol{\Phi}$ is set equal to the identity matrix. Expand the variance function $\boldsymbol{\Sigma}_{i}$ so that

$$
\operatorname{Var}\left(\varepsilon_{i}\right)=\boldsymbol{\Phi}^{\frac{1}{2}} \boldsymbol{A}_{i}^{\frac{1}{2}} \boldsymbol{R}_{i} \boldsymbol{A}_{i}^{\frac{1}{2}} \boldsymbol{\Phi}^{\frac{1}{2}},
$$

where $\boldsymbol{R}_{i}$ is the correlation matrix and $\boldsymbol{A}_{i}$ is a diagonal matrix containing the variances following from the generalized linear model specification of $\boldsymbol{Y}_{i j}$ given the random effects $\boldsymbol{b}_{i}=\mathbf{0}$, i.e., with diagonal elements $v\left(\mu_{i j} \mid \boldsymbol{b}_{i}=\mathbf{0}\right)$. Using (F.3) and (F.5), we have the following expression for the variance-covariance matrix (F.1):

$$
\boldsymbol{V}_{i} \cong \boldsymbol{\Delta}_{i} \boldsymbol{Z}_{i} \boldsymbol{D} \boldsymbol{Z}_{i}^{\prime} \boldsymbol{\Delta}_{i}^{\prime}+\boldsymbol{\Phi}^{\frac{1}{2}} \boldsymbol{A}_{i}^{\frac{1}{2}} \boldsymbol{R}_{i} \boldsymbol{A}_{i}^{\frac{1}{2}} \boldsymbol{\Phi}^{\frac{1}{2}} .
$$

When the canonical link is used, we have $\boldsymbol{A}_{i}=\boldsymbol{\Delta}_{i}$ and (F.6) can be written as: $\boldsymbol{V}_{i} \cong \boldsymbol{\Delta}_{i} \boldsymbol{Z}_{i} \boldsymbol{D} \boldsymbol{Z}_{i}^{\prime} \boldsymbol{\Delta}_{i}^{\prime}+$ $\boldsymbol{\Phi}^{\frac{1}{2}} \boldsymbol{\Delta}_{i}^{\frac{1}{2}} \boldsymbol{R}_{i} \boldsymbol{\Delta}_{i}^{\frac{1}{2}} \boldsymbol{\Phi}^{\frac{1}{2}}$. If in addition, conditional independence (no serial correlation) is assumed, then (F.6) simplifies to: $\boldsymbol{V}_{i} \cong \boldsymbol{\Delta}_{i} \boldsymbol{Z}_{i} \boldsymbol{D} \boldsymbol{Z}_{i}^{\prime} \boldsymbol{\Delta}_{i}^{\prime}+\boldsymbol{\Phi}^{\frac{1}{2}} \boldsymbol{\Delta}_{i} \boldsymbol{\Phi}^{\frac{1}{2}}$. Further, if we reduce the random-effects part to a random-intercept model, i.e., $\boldsymbol{Z}_{i}=\mathbf{1}$ and $\boldsymbol{D}=d$, then (F.6) reduces to $\boldsymbol{V}_{i} \cong \boldsymbol{\Delta}_{i}(d \boldsymbol{J}) \boldsymbol{\Delta}_{i}^{\prime}+$ $\Phi^{\frac{1}{2}} \boldsymbol{\Delta}_{i} \boldsymbol{\Phi}^{\frac{1}{2}}$. The result is particularly simple to use in the case of normal outcomes of course. Vangeneugden et al (2008b) applied the result to binary data and zoomed in on the random-intercept setting. This will be reviewed in Section F.3, after which we turn to the count-data case in Section F.2.

\section{F.2 Approximate Forms for the Poisson Case}

Focusing on the random-intercepts only case, we can write for the count-data case:

$$
\boldsymbol{Y}_{i}=\boldsymbol{\mu}_{i}+\boldsymbol{\varepsilon}_{i}=e^{X_{i} \boldsymbol{\xi}_{+} \boldsymbol{J}_{i} b_{i}}+\boldsymbol{\varepsilon}_{i},
$$

where $\boldsymbol{J}_{i}$ is an $n_{i}$-dimensional vector of ones, and further assuming that there is no overdispersion, i.e., $\Phi=I$, it follows that

$$
\begin{aligned}
& v_{i j j}=e^{\boldsymbol{x}_{i j}^{\prime} \boldsymbol{\xi}}\left(d e^{\boldsymbol{x}_{i j}^{\prime} \boldsymbol{\xi}}+1\right),
\end{aligned}
$$

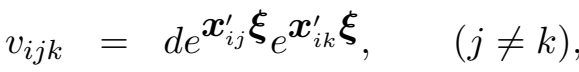

producing the correlation-approximation:

$$
\rho_{i j k} \approx \frac{d e^{\boldsymbol{x}_{i j}^{\prime} \boldsymbol{\xi}} e^{\boldsymbol{x}_{i k}^{\prime} \boldsymbol{\xi}}}{\sqrt{e^{\boldsymbol{x}_{i j}^{\prime} \boldsymbol{\xi}}\left(d e^{\boldsymbol{x}_{i j}^{\prime} \boldsymbol{\xi}}+1\right) \cdot e^{\boldsymbol{x}_{i k}^{\prime} \boldsymbol{\xi}}\left(d e^{\boldsymbol{x}_{i k}^{\prime} \boldsymbol{\xi}}+1\right)}} .
$$

Note that, also here, (F.7) reduces to zero correlation for $d=0$, and that $\rho_{i j k} \rightarrow 1$ when $d \rightarrow \infty$. Further assuming exchangeability, i.e., $\boldsymbol{x}_{i j}^{\prime} \boldsymbol{\xi}=\xi$, (F.7) simplifies to

$$
\rho \approx \frac{d e^{\xi}}{1+d e^{\xi}} .
$$




\section{Beta $=-1$}

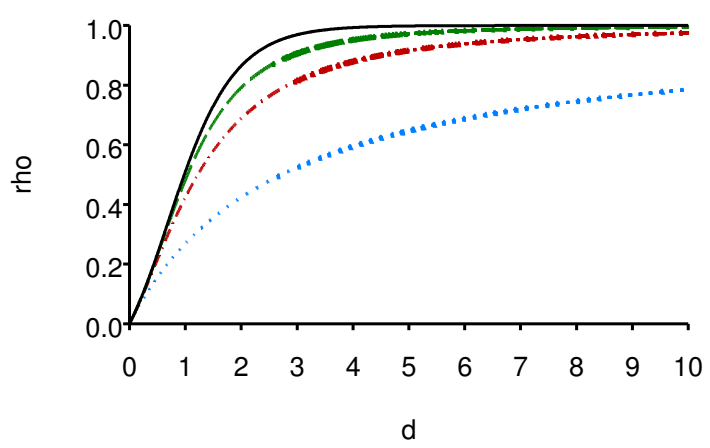

Beta $=1$
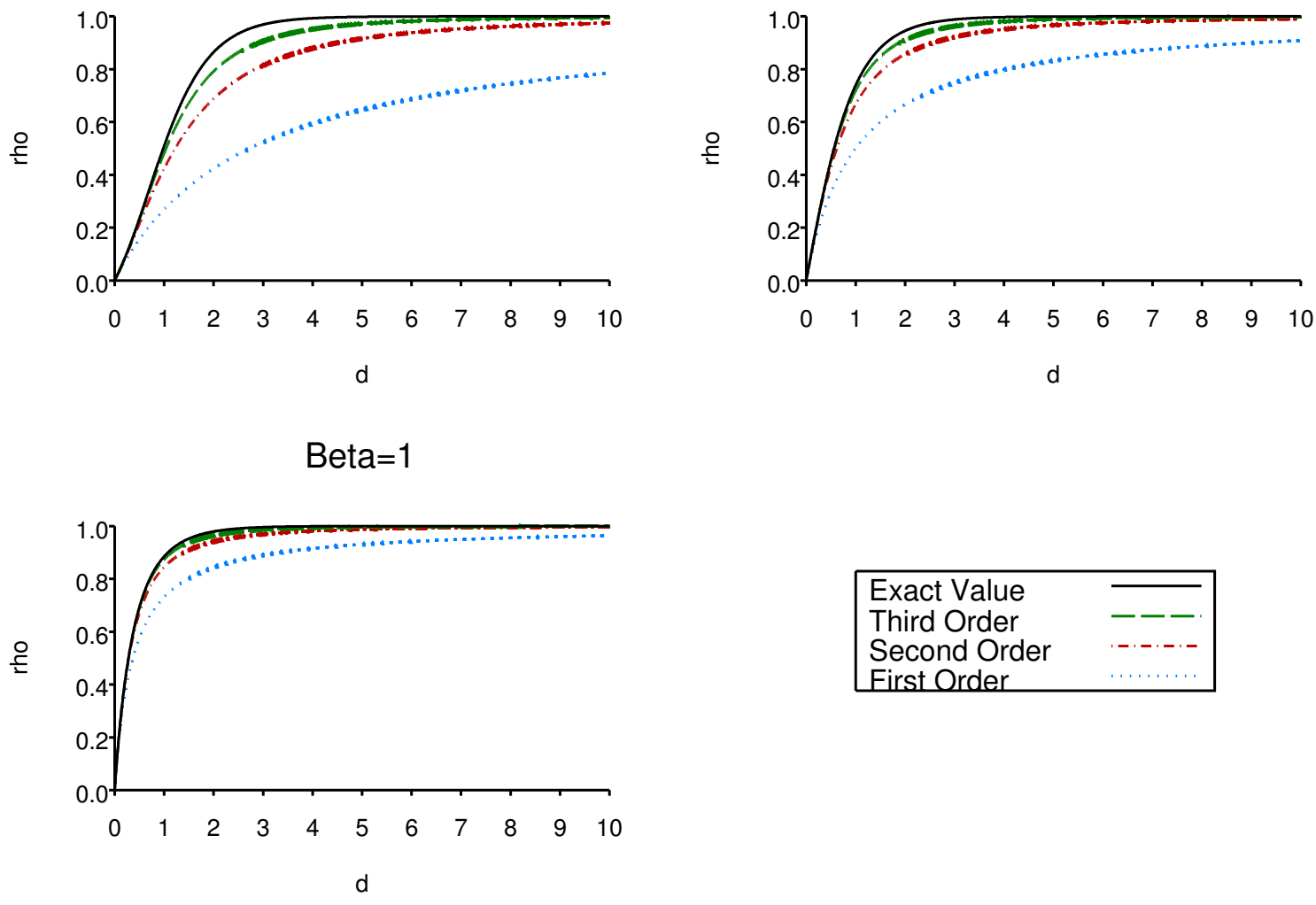

Figure 1: Poisson model. Quality of Taylor-series approximations for the correlation function in the clustered exchangeable case.

Evidently, we could also start from the explicit expression (F.20) and derive, by Taylor-series expansions for numerator and denominator separately:

$$
\rho=\left[e^{\xi} \sum_{n=1}^{+\infty}\left(\frac{3^{n}-1}{n ! 2^{n}}\right) d^{n}\right] /\left[1+e^{\xi} \sum_{n=1}^{+\infty}\left(\frac{3^{n}-1}{n ! 2^{n}}\right) d^{n}\right]
$$

Obviously, (F.7) immediately follows from (F.8) by restricting the Taylor series to the first order. To get a rough idea of the approximations' quality, assume $\xi=0.6236$ and $d=1.1792$, then $\rho=\rho_{[\infty]}=$ 0.8834 . The first order approximation is $\rho_{[1]}=0.6875$, with subsequent values $\rho_{[2]}=0.8274$ and $\rho_{[3]}=0.8658$. The eighth order is accurate to four decimal places while, if eight correct decimals are required, one has to go up to order 13 . Figure 1 presents the quality of the first- to third-order approximations, for $\xi=-1,0$, and 1 , and for the range $[0,10]$ for $d$. Apart from the obvious increase in quality with increasing order, it is also clear that all orders converge rather quickly to the correct value with increasing random-intercept variance. Given that the numerator and denominator of (F.9) differ only in the constant term, this is not surprising, since for increasing $d$ the leading terms, will rapidly dominate.

Switching to the combined model for the exchangeable case, the explicit form for the correlation 
becomes:

$$
\rho=\frac{\phi\left(e^{\xi+\frac{1}{2} d}\right)\left[\left(\frac{\tau \sigma^{2}}{\phi^{2}}+1\right) e^{d}-1\right]}{1+\phi\left(e^{\xi+\frac{1}{2} d}\right)\left[\left(\frac{\sigma^{2}}{\phi^{2}}+1\right) e^{d}-1\right]} .
$$

Similarly, a Taylor series expansion is:

$$
\rho=\left[\phi e^{\xi} \sum_{n=1}^{+\infty}\left(\frac{3^{n} \tau \sigma^{2}+\left(3^{n}-1\right) \phi^{2}}{n ! 2^{n} \phi^{2}}\right) d^{n}\right] /\left[1+\phi e^{\xi} \sum_{n=1}^{+\infty}\left(\frac{3^{n} \sigma^{2}+\left(3^{n}-1\right) \phi^{2}}{n ! 2^{n} \phi^{2}}\right) d^{n}\right] .
$$

\section{F.3 Approximate Forms for the Binary Case}

There is a long tradition for the derivation of the intraclass correlation with binary data (Ridout, Demétrio, and Firth 1999). This case is both relevant and evasive, because the popular logit-normal one eludes a closed-form, owing to the absence of strong conjugacy in this case. Of course, the probit model is a different matter and we will return to this in Section F.6.

As a basis for comparison with the Poisson case to be dealt with next, let us first review Vangeneugden et al (2008b)'s derivation of the ICC for a random-intercept model for binomial data with a logit link and assuming no overdispersion. In this case, $\boldsymbol{V}_{i}$ reduces to $\boldsymbol{V}_{i} \cong \boldsymbol{\Delta}_{i}(d \boldsymbol{J}) \boldsymbol{\Delta}_{i}^{\prime}+\boldsymbol{\Delta}_{i}=$ $\boldsymbol{\Delta}_{i}\left(d \boldsymbol{J}+\boldsymbol{\Delta}_{i}^{-1}\right) \boldsymbol{\Delta}_{i}^{\prime}$. Furthermore, $\boldsymbol{\Delta}_{i}$ is a diagonal matrix with $V_{i j}(0)$ as diagonal elements, where the variance function $V_{i j}(0)=\left.\mu_{i j}\right|_{\boldsymbol{b}_{i}=0}\left(1-\left.\mu_{i j}\right|_{\boldsymbol{b}_{i}=0}\right)$, and therefore $\boldsymbol{V}_{i} \cong \operatorname{diag}\left(V_{i j}(0)\right)[d \boldsymbol{J}+$ $\left.\operatorname{diag}\left(V_{i j}(0)\right)^{-1}\right] \operatorname{diag}\left(V_{i j}(0)\right)$. In other words, the variance-covariance matrix for subject $i$ is specified by the matrix with elements: $v_{i j j}=V_{i j}(0)\left[1+V_{i j}(0) d\right], v_{i j k}=d V_{i j}(0) V_{i k}(0), \quad(j \neq k)$. Based on these, we can determine a first-order approximation of the marginal correlation between time point $j$ and $k$, which is the intraclass correlation coefficient of reliability:

$$
\rho_{i j k}=\operatorname{Corr}\left(Y_{i j}, Y_{i k}\right)=\frac{V_{i j}(0) V_{i k}(0) d}{\sqrt{\left\{V_{i j}(0)\left[1+V_{i j}(0) d\right]\right\}\left\{V_{i k}(0)\left[1+V_{i k}(0) d\right]\right\}}} .
$$

Note that, when $d=0$, then $\rho_{i j k}=0$, and when $d \rightarrow \infty$, then $\rho_{i j k} \rightarrow 1$. Thus, the full positive correlation range is attainable, quite unlike marginal models for correlated binary data, that experience restrictions on the correlation parameter space to certain degrees. For a discussion, see Molenberghs and Verbeke (2005). No negative correlations can occur, which is entirely in line with the model's hierarchical nature, i.e., where $d$ is and remains interpretable as a variance. The related discussion for the case of linear mixed models can be consulted in Verbeke and Molenberghs (2000).

\section{F.4 Closed-form Expressions}

Whenever the marginal joint distribution can be derived, in particular, when the univariate and bivariate moments can be calculated explicitly, then the marginal correlation can simply be derived therefrom. This is true for the normal, Poisson, probit, and Weibull cases as studied before. As always, the logit is the notorious exception.

Generically, strong conjugacy is a sufficient condition for the existence of such closed forms. Note that this is not necessary, the probit model providing an important counterexample.

These considerations are not particular to the correlation coefficient, but apply to every association measure (e.g., the odds ratio) that is a simple analytical function of the marginal moments. 


\section{F.5 Closed Forms for the Poisson Case}

As stated in the previous section, Molenberghs, Verbeke, and Demétrio (2007) derived closed-form mean (B.1) and variance (B.15) for the combined model in the general, longitudinal context. These produce, as special cases, expressions for the negative binomial and the Poisson-normal models. Variance-covariance expression (B.15) renders straightforward the derivation of a closed-form correlation function expression.

In the general case of the combined model for longitudinal data with arbitrary fixed- and randomeffects structures, the variance, deriving from (B.15) equals:

$$
\operatorname{Var}\left(Y_{i j}\right)=\phi_{i j} e^{\boldsymbol{x}_{i j}^{\prime} \boldsymbol{\xi}+\frac{1}{2} \boldsymbol{z}_{i j} D \boldsymbol{z}_{i j}^{\prime}}+\sigma_{i, j j} e^{2 \boldsymbol{x}_{i j}^{\prime} \boldsymbol{\xi}+2 \boldsymbol{z}_{i j} D \boldsymbol{z}_{i j}^{\prime}}+\phi_{i j}^{2} e^{2 \boldsymbol{x}_{i j}^{\prime} \boldsymbol{\xi}+\boldsymbol{z}_{i j} D \boldsymbol{z}_{i j}^{\prime}}\left(e^{\boldsymbol{z}_{i j} D \boldsymbol{z}_{i j}^{\prime}}-1\right) .
$$

Likewise, the covariance can be written as:

$$
\begin{aligned}
\operatorname{Cov}\left(Y_{i j}, Y_{i k}\right)= & \phi_{i j} e^{\boldsymbol{x}_{i j}^{\prime} \boldsymbol{\xi}+\frac{1}{2} \boldsymbol{z}_{i j} D \boldsymbol{z}_{i j}^{\prime}}\left[\left(\frac{\sigma_{i, j k}}{\phi_{i j} \phi_{i k}}+1\right) e^{\frac{1}{2}\left(\boldsymbol{z}_{i j} D \boldsymbol{z}_{i k}^{\prime}+\boldsymbol{z}_{i k} D \boldsymbol{z}_{i j}^{\prime}\right)}-1\right] \times \\
& \times \phi_{i k} e^{\boldsymbol{x}_{i k}^{\prime} \boldsymbol{\xi}+\frac{1}{2} \boldsymbol{z}_{i k} D \boldsymbol{z}_{i k}^{\prime}} .
\end{aligned}
$$

The correlation between two measurements $j$ and $k$ on the same experimental unit $i$ then is:

$$
\operatorname{Corr}\left(Y_{i j}, Y_{i k}\right)=\frac{\operatorname{Cov}\left(Y_{i j}, Y_{i k}\right)}{\sqrt{\operatorname{Var}\left(Y_{i j}\right) \cdot \operatorname{Var}\left(Y_{i k}\right)}} .
$$

Because of its generality, it is hard to simplify (F.13), unless in specific cases. Of course, (F.11) and (F.12) simplify when we zoom in on the Poisson-normal case:

$$
\begin{aligned}
\operatorname{Var}\left(Y_{i j}\right) & =e^{\boldsymbol{x}_{i j}^{\prime} \boldsymbol{\xi}+\frac{1}{2} \boldsymbol{z}_{i j} D \boldsymbol{z}_{i j}^{\prime}}+e^{2 \boldsymbol{x}_{i j}^{\prime} \boldsymbol{\xi}+\boldsymbol{z}_{i j} D \boldsymbol{z}_{i j}^{\prime}}\left(e^{\boldsymbol{z}_{i j} D \boldsymbol{z}_{i j}^{\prime}}-1\right), \\
\operatorname{Cov}\left(Y_{i j}, Y_{i k}\right) & =e^{\boldsymbol{x}_{i j}^{\prime} \boldsymbol{\xi}+\frac{1}{2} \boldsymbol{z}_{i j} D \boldsymbol{z}_{i j}^{\prime}}\left(e^{\boldsymbol{z}_{i j} D \boldsymbol{z}_{i k}^{\prime}}-1\right) e^{\boldsymbol{x}_{i k}^{\prime} \boldsymbol{\xi}+\frac{1}{2} \boldsymbol{z}_{i k} D \boldsymbol{z}_{i k}^{\prime}}
\end{aligned}
$$

Likewise, they do for the negative-binomial case. There are two ways to approach this case. First, one can absorb the fixed effects into $\theta_{i j}$ and hence the conventional expression follows. However, let us opt for the second route, where (22) is maintained, only with $\boldsymbol{b}_{\boldsymbol{i}}$ removed or, equivalently, $D=0$. Alternatively, we can start from (F.11) and (F.12), of course. At any rate, the variance and covariance can be written as:

$$
\begin{aligned}
\operatorname{Var}\left(Y_{i j}\right) & =\phi_{i j} \mu_{i j}+\sigma_{i, j j} \mu_{i j}^{2}, \\
\operatorname{Cov}\left(Y_{i j}, Y_{i k}\right) & =\mu_{i j} \mu_{i k} \sigma_{i, j k} .
\end{aligned}
$$

Here, $\mu_{i j}=\exp \left(\boldsymbol{x}_{i j}^{\prime} \boldsymbol{\xi}\right)$. Evidently, (F.13) can be written in a convenient form as:

$$
\operatorname{Corr}\left(Y_{i j}, Y_{i k}\right)=\frac{\mu_{i j} \mu_{i k} \sigma_{i, j k}}{\sqrt{\left(\phi_{i j} \mu_{i j}+\mu_{i j}^{2} \sigma_{i, j j}\right) \cdot\left(\phi_{i k} \mu_{i k}+\mu_{i k}^{2} \sigma_{i, k k}\right)}} .
$$

Note that, when the fixed effects are subsumed into $\theta_{i j}$, the variance is written as $\mu_{i j}+\sigma_{i, j j}$ with covariance $\sigma_{i, j k}$, in which case the alternative, simple and conventional form for the correlation results:

$$
\operatorname{Corr}\left(Y_{i j}, Y_{i k}\right)=\frac{\sigma_{i, j k}}{\sqrt{\left(\mu_{i j}+\sigma_{i, j j}\right) \cdot\left(\mu_{i k}+\sigma_{i, k k}\right)}}
$$


Additional insight can be obtained for special but important cases resulting from simplifying the mean and variance structures of the models. Let us show this for an exchangeable structure, where $\boldsymbol{x}_{i j}^{\prime} \boldsymbol{\xi}=\xi, \boldsymbol{z}_{i j}=1$, and $D=d$. It is then sensible to also set $\phi_{i j}=\phi, \sigma_{i, j j}=\sigma^{2}$, and $\sigma_{i, j k}=\sigma^{2} \tau$, for $j \neq k$. For the combined model, we obtain the following simplifications of (F.11) and (F.12):

$$
\begin{aligned}
\operatorname{Var}\left(Y_{i j}\right) & =\phi e^{\xi+\frac{1}{2} d}+\phi^{2}\left(e^{\xi+\frac{1}{2} d}\right)^{2} \cdot\left[\left(\frac{\sigma^{2}}{\phi^{2}}+1\right) e^{d}-1\right], \\
\operatorname{Cov}\left(Y_{i j}, Y_{i k}\right) & =\phi^{2}\left(e^{\xi+\frac{1}{2} d}\right)^{2} \cdot\left[\left(\frac{\tau \sigma^{2}}{\phi^{2}}+1\right) e^{d}-1\right] .
\end{aligned}
$$

As a result, the correlation becomes:

$$
\operatorname{Corr}\left(Y_{i j}, Y_{i k}\right)=\frac{\phi e^{\xi+\frac{1}{2} d}\left[\left(\frac{\tau \sigma^{2}}{\phi^{2}}+1\right) e^{d}-1\right]}{1+\phi e^{\xi+\frac{1}{2} d} \cdot\left[\left(\frac{\sigma^{2}}{\phi^{2}}+1\right) e^{d}-1\right]} .
$$

Considering the special case with only normally distributed random-effects, i.e., an exchangeable version of the conventional Poisson-normal model $\left(\phi_{i j} \equiv 1\right.$ and $\left.\sigma^{2} \equiv 0\right)$, simple algebra leads to:

$$
\operatorname{Corr}\left(Y_{i j}, Y_{i k}\right)=\frac{e^{\xi+\frac{1}{2} d}\left(e^{d}-1\right)}{1+e^{\xi+\frac{1}{2} d}\left(e^{d}-1\right)} .
$$

On the other hand, assuming that only the gamma-type random effects are present $(d=0)$, we derive:

$$
\operatorname{Corr}\left(Y_{i j}, Y_{i k}\right)=\frac{\mu^{2} \tau \sigma^{2}}{\phi \mu+\sigma^{2} \mu^{2}}=\frac{\tau \operatorname{Var}(\lambda)}{E(\lambda)+\operatorname{Var}(\lambda)}
$$

where $\lambda=\phi \mu=\phi e^{\xi}$.

\section{F.6 Closed Forms for the Binary Case}

Given the availability of closed-form moments, and hence variances (D.5) and covariances (D.6), the correlation merely follows from (F.13) . Observe that the probit-based correlated is also of relevance to the logit case, given approximate relationship (37) and correspondences derived therefrom. Such marginalization considerations can also be found in Skrondal and Rabe-Hesketh (2004, p. 124), both for the probit as well as for the logit model.

\section{F.6.1 The Odds Ratio}

The marginal odds ratio is defined as

$$
\mathrm{OR}_{i, j k}=\frac{P\left(Y_{i j}=1, Y_{i k}=1\right) \cdot P\left(Y_{i j}=0, Y_{i k}=0\right)}{P\left(Y_{i j}=1, Y_{i k}=0\right) \cdot P\left(Y_{i j}=0, Y_{i k}=1\right)} .
$$

In the probit case, the factors in (F.21) can be calculated from (35), for $n_{i}=2$ and $n_{i}=1$, and combination formula (D.3).

Should the logit model be used directly, then the approximations, laid out in Section 4.2, and applied to the binary case in Section F.3, have to be used. 


\section{F.7 Closed Forms for the Time-to-Event Case}

Also here, the marginal correlation is easy to calculate, using the expression for the probit case, but then based on (E.14) and (E.15). 